\title{
Sulfur volatiles from Allium spp. affect Asian citrus psyllid, Diaphorina citri Kuwayama (Hemiptera: Psyllidae), response to citrus volatiles
}

\author{
R.S. Mann, R.L. Rouseff, J.M. Smoot, W.S. Castle \\ and L.L. Stelinski* \\ Citrus Research and Education Center, University of Florida, 700 \\ Experiment Station Road, Lake Alfred, FL 33850, USA
}

\begin{abstract}
The Asian citrus psyllid, Diaphorina citri Kuwayama, vectors Candidatus Liberibacter asiaticus (Las) and Candidatus Liberibacter americanus (Lam), the presumed causal agents of huanglongbing. D. citri generally rely on olfaction and vision for detection of host cues. Plant volatiles from Allium spp. (Alliaceae) are known to repel several arthropod species. We examined the effect of garlic chive (A. tuberosum Rottl.) and wild onion (A. canadense L.) volatiles on D. citri behaviour in a two-port divided T-olfactometer. Citrus leaf volatiles attracted significantly more $D$. citri adults than clean air. Volatiles from crushed garlic chive leaves, garlic chive essential oil, garlic chive plants, wild onion plants and crushed wild onion leaves all repelled $D$. citri adults when compared with clean air, with the first two being significantly more repellent than the others. However, when tested with citrus volatiles, only crushed garlic chive leaves and garlic chive essential oil were repellent, and crushed wild onions leaves were not.

Analysis of the headspace components of crushed garlic chive leaves and garlic chive essential oil by gas chromatography-mass spectrometry revealed that monosulfides, disulfides and trisulfides were the primary sulfur volatiles present. In general, trisulfides (dimethyl trisulfide) inhibited the response of D. citri to citrus volatiles more than disulfides (dimethyl disulfide, allyl methyl disulfide, allyl disulfide). Monosulfides did not affect the behaviour of D. citri adults. A blend of dimethyl trisulfide and dimethyl disulfide in $1: 1$ ratio showed an additive effect on inhibition of $D$. citri response to citrus volatiles. The plant volatiles from Allium spp. did not affect the behaviour of the D. citri ecto-parasitoid Tamarixia radiata (Waterston). Thus, Allium spp. or the tri- and di-sulphides could be integrated into management programmes for D. citri without affecting natural enemies.
\end{abstract}

Keywords: Diaphorina citri, Tamarixia radiata, garlic chive, dimethyl trisulfide, dimethyl disulfide, citrus

(Accepted 3 April 2010)

*Author for correspondence

Fax: 863-956-4631

E-mail: stelinski@ufl.edu 


\section{Introduction}

Asian citrus psyllid, Diaphorina citri Kuwayama (Hemiptera: Psyllidae), is one of the most serious pests of citrus worldwide because it vectors the bacterial pathogen causing huanglongbing (HLB) disease. In addition, both nymphs and adults are obligate phloem feeders that cause chlorosis on infested leaves and excrete honeydew that promotes the growth of sooty mold. HLB affects plant phloem, causing yellow shoots, mottling, chlorosis and twig die back, which cause rapid tree decline and may ultimately cause tree death. Fruit on diseased trees do not colour properly and can be bitter tasting and misshapen as well as reduced in size (Capoor, 1963; Halbert \& Manjunath, 2004; Bové, 2006). Where HLB is present, infected citrus trees decline and may die within a few years and never produce usable fruit (Halbert \& Manjunath, 2004). The disease is presumed to be caused by either of three species of phloem-limited, noncultured, Gram-negative bacteria: Candidatus Liberibacter asiaticus (Las), Candidatus Liberibacter africanus (Laf) and Candidatus Liberibacter americanus (Lam) (Bové, 2006). The bacteria are efficiently vectored by two species of citrus psyllid (D. citri and Trioza erytreae (del Guercio)).

Direct damage caused by psyllid feeding is restricted to young and tender leaves; therefore, control measures were previously only justified for non-bearing citrus which often produce shoot flushes throughout the growing season. The discovery of HLB disease in Florida in 2005 changed the status of $D$. citri to a major pest requiring management in all stages of citrus production (Hodges \& Morse, 2009). Currently, a broad range of vector and disease control methods is being employed to control this disease in Florida (Qureshi \& Stansly, 2007; Rogers \& Timmer, 2007). Current management practices are incomplete because of a lack of known resistant cultivars (Halbert \& Manjunath, 2004), effective biological control agents (Qureshi \& Stansly, 2007) and cultural control options (Childers \& Rogers, 2005; Powell et al., 2007). Classical biological control of D. citri with the introduced parasitoid, Tamarixia radiata (Waterston), was initiated in Florida in 1999 (Hoy \& Nguyen, 2001). The parasitoid established and dispersed quickly, but parasitism rates are generally low and variable (Qureshi et al., 2009). Primary control of the vector has relied on broad spectrum insecticides (Rogers, 2008). However, insecticide use is known to negatively affect populations of natural enemies and may lead to development of insecticide resistance. Therefore, novel management strategies, such as insect repellents, attractants and antifeedents, may serve as useful alternatives or supplements to insecticides.

All known host plants of $D$. citri belong to the family Rutaceae and comprise a wide range of species including ten from genera other than Citrus (Aubert, 1990). D. citri generally rely on olfaction and vision for detection of host cues (Moran \& Brown, 1973; Kristoffersen et al., 2006; Onagbola et al., 2009; Wenninger et al., 2009). Interplanting guava, Psidium guajava (L.), has been reported to reduce D. citri populations in citrus orchards in Vietnam, possibly due to volatile chemicals (Beattie et al., 2006; Hall et al., 2008; Zaka et al., 2010). Recently, Rouseff et al. (2008) identified dimethyl disulfide (DMDS) from guava as a possible defensive volatile that may explain guava's repellency to $D$. citri. Plants in the family Alliaceae also have been shown to produce sulfur compounds known to repel arthropods (Auger et al., 1989; Dugravot et al., 2002, 2003, 2004,
2005; Amarawardana et al., 2007). Chemicals from Allium sativum (L.) have been reported to repel cotton bollworms (Gurusubramanian \& Krishna, 1996), codling moth (Landolt et al., 1999), aphids (Assis et al., 2007), whiteflies (Salas, 2001), cabbage root flies (Prowse et al., 2006), mosquitoes (Snow \& Cutler, 2006) and phlebotomine sand flies (Valerio \& Maroli, 2005). Furthermore, garlic chive, A. tuberosum, has been shown to repel green peach aphid, Myzus persicae (Sulzer), in sweet pepper (Amarawardana et al., 2007). In addition, the leek, A. porrum L., has been reported to produce sulfur-based chemicals in response to Acrolepiopsis assectella (Zeller) attack (Dugravot et al., 2002, 2005). Based on the repellent properties of these plants, certain Allium spp. products such as garlic barrier ${ }^{\circledR}$ (Garlic Research Labs, CA), Garlic shield ${ }^{\circledR}$ (Nutritek Inc., OR) and Biorepel ${ }^{\mathbb{R}}$ (JH Biotech Inc., CA) have been commercially formulated for pest management. In this series of experiments, we examined the effect of Allium spp. as well as sulfur volatiles identified from them on the behaviour of $D$. citri and its parasitoid, T. radiata. Our objective is to identify possible effective repellents for $D$. citri that have minimal effects on its parasitoid.

\section{Methods and materials}

Insects

Adult D. citri used in behavioural bioassays were obtained from a laboratory culture at the University of Florida Citrus Research and Education Center (Lake Alfred, USA). The culture was established in 2000 from field populations in Polk Co., FL, USA $\left(28.0^{\prime} \mathrm{N}, 81.9^{\prime} \mathrm{W}\right)$ prior to the discovery of HLB in FL. The culture is maintained without exposure to insecticides on sour orange (Citrus aurantium L.) and 'Hamlin' orange ((C. sinensis (L.) Osb.) seedlings at $27 \pm 1^{\circ} \mathrm{C}, 63 \pm 2 \% \mathrm{RH}$ and under a L14:D10 photoperiod. The sexual maturity of psyllids was confirmed by placing adults for at least seven days on citrus seedlings in Plexiglass ${ }^{\circledR}$ cages prior to use in experiments (Wenninger \& Hall, 2007). Tamarixia radiata adults were collected from newly expanded 'Hamlin' orange leaf flush infested with $D$. citri from mature trees in an unsprayed grove in Polk Co., FL. The flush was maintained in $40 \times 40 \times 40 \mathrm{~cm}$ Plexiglas cages at $26 \pm 1^{\circ} \mathrm{C}, 60 \pm 5 \% \mathrm{RH}$ and under a photoperiod of 14:10 (L:D) h. Parasitoids emerging from psyllid nymphs were collected daily and maintained as described in Onagbola et al. (2009).

\section{Plant material and chemical samples}

Garlic chive (A. tuberosum), wild onion (A. canadense), white guava ( $P$. guajava) and Valencia orange (C. sinensis) plants were grown in 1.0 gallon pots in a temperature controlled green house. Allium spp. samples were composed of 10-week-old whole plants. Since fresh leaves are known to contain higher proportions of secondary compounds (Hrutfiord et al., 1974) and D. citri are exclusively associated with new growth (Catling, 1970), we harvested approximately $2.0 \mathrm{~g}$ of fresh leaf flush (immature leaves of the growing shoots (Hall \& Albrigo, 2007)) for D. citri behavioural assays. Dimethyl disulfide (DMDS) ( $\geqslant 98 \%$ purity), dimethyl trisulfide (DMTS) ( $\geqslant 98.5 \%$ purity), allyl methyl sulfide (AMS) $(\geqslant 98 \%$ purity) and Chinese garlic chive essential oil (unknown purity) were obtained from SigmaAldrich Inc. USA. Allyl methyl disulfide (AMDS) $(\geqslant 98 \%$ 


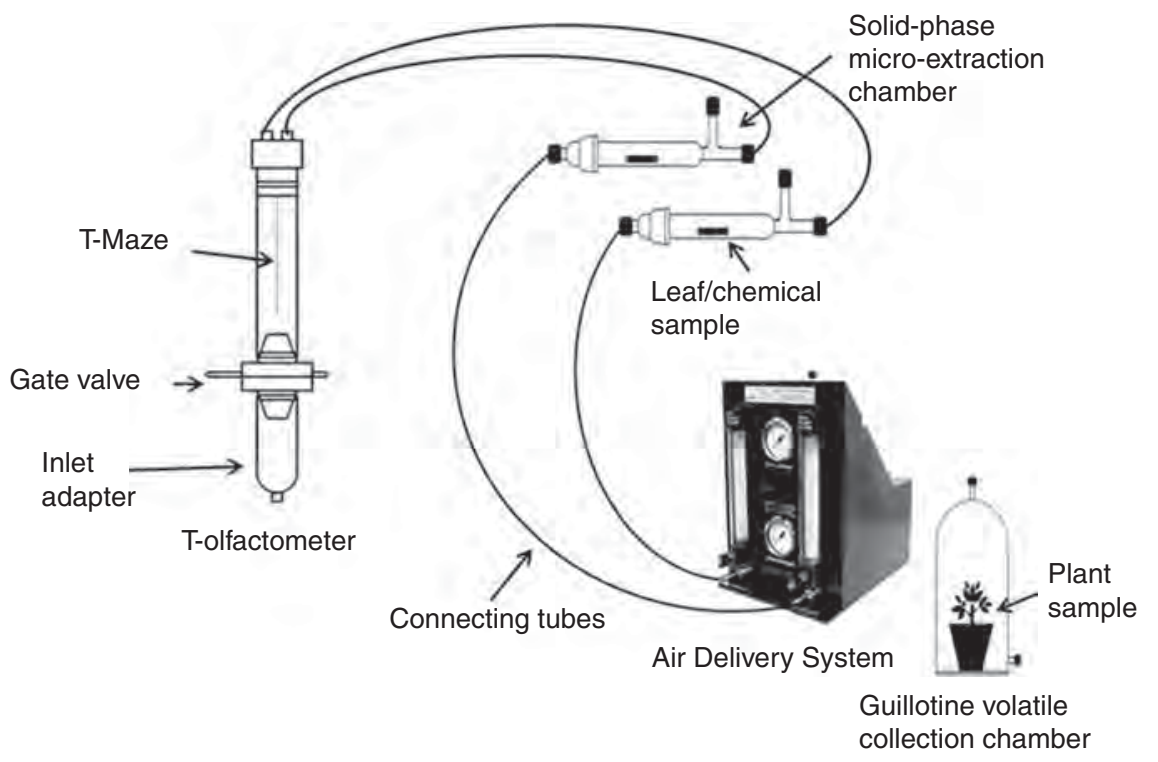

Fig. 1. Schematic diagram of the T-maze olfactometer used for behavioural bioassays. The guillotine volatile collection chambers were replaced with the solid-phase micro-extraction chambers for leaf samples.

purity) and allyl disulfide (ADS) ( $\sim 80.0 \%$ purity) were obtained from Frutarom (UK) Ltd, UK and Penta Chemical Company, USA, respectively.

\section{Behavioural bioassays with plant material}

A custom designed two-port divided T-olfactometer (fig. 1) from Analytical Research Systems (ARS) Inc., Gainesville, FL was used to evaluate behavioural response of D. citri to Allium spp. plants. The olfactometer consisted of a $30 \mathrm{~cm}$ long glass tube with $3.5 \mathrm{~cm}$ internal diameter that is bifurcated into two equal halves with a Teflon strip forming a T-maze. Each half served as an arm of the olfactometer, enabling the $D$. citri to make a choice between two potential odour fields. The olfactometer arms were connected to odour sources placed in guillotine volatile collection chambers or solid-phase micro-extraction chambers (ARS, Gainesville, FL) through Teflon ${ }^{\circledR}$ glass tube connectors (fig. 1). The plant samples were placed directly in 51 guillotine volatile collection chambers (GVCC) (fig. 1). For plant samples, a $35 \mathrm{~cm}$ tall $\times 15 \mathrm{~cm}$ wide dome shaped GVCC was placed directly over the pots. The GVCC was supported with a Teflon guillotine support base (GSB) (ARS, Gainesville, FL) (fig. 1). The GVCC was attached with an inlet valve on the side of a chamber and an outlet valve on top of the dome for incoming and outgoing air streams, respectively. The leaf samples were placed in solid-phase micro-extraction chambers (SPMEC) (ARS, Gainesville, FL). The SPMEC consists of a straight glass tube $(17.5 \mathrm{~cm}$ long $\times 2.5 \mathrm{~cm}$ internal diameter supported with an inlet and outlet valve for incoming and outgoing air streams, respectively. The leaf samples were wrapped in laboratory tissue (Kimwipes, Kimberly-Clark, Roswell, GA) to minimize contamination of the SPMEC. The purified and humidified air was pushed through the GVCC or SPMEC via two pumps connected to an air delivery system (ARS, Gainesville, FL) (fig. 1). A constant airflow of $0.11 \mathrm{~min}^{-1}$ was maintained through both arms of the olfactometer. Purified and humidified air was pushed through these chambers via two pumps connected to an air delivery system (ARS, Gainesville, FL) (fig. 1). The olfactometer was housed within a temperature controlled room and positioned vertically under a fluorescent 900 lux light bulb positioned within a $1.0 \times 0.6 \times 0.6 \mathrm{~m}$ fibre board box for uniform light diffusion. This position took advantage of the negative geotactic and positive phototactic response of D. citri (Mann et al., unpublished results). The olfactometer inlet adapter was covered with black cloth to facilitate insect movement towards odour sources. An odour source was randomly assigned to one of the arms of the olfactometer at the beginning of each bioassay and was reversed after every 30 insects to eliminate positional bias.

Female D. citri are more responsive to citrus odours than males (Wenninger et al., 2009); therefore, only female adults were tested in this study. Prior to tests involving putative repellents, $D$. citri female adults were exposed to clean air vs. clean air and citrus vs. citrus in the T-maze olfactometer to verify the absence of positional bias. Thereafter, the following odour source combinations were tested: (i) garlic chive plant vs. clean air; (ii) crushed garlic chive leaves vs. clean air; (iii) wild onion plant vs. clean air; (iv) crushed wild onion leaves vs. clean air; (v) garlic chive essential oil vs. clean air; (vi) citrus leaves vs. clean air; and (vii) clean air vs. clean air (control). The putative repellents were also evaluated in combination with citrus odours to verify their repellent effect in presence of citrus odours. The treatment tested for this set of experiments were: (i) crushed garlic chive leaves + citrus leaves vs. citrus leaves; (ii) crushed wild onion leaves + citrus leaves vs. citrus leaves; (iii) garlic chive essential oil + citrus leaves vs. citrus leaves; (iv) clean air vs. clean air (negative control); and (v) citrus leaves vs. citrus leaves (positive control). The plant samples were composed of 10-week-old whole plants, while leaf samples were composed of $\sim 2.0 \mathrm{~g}$ of fresh leaves obtained from Allium or citrus plants. A minimum of 120 D. citri female adults were examined per treatment combination (four replications of $30 \mathrm{D}$. citri per replication). D. citri females were released 
individually into the inlet adapter at the base of the olfactometer. Adults were given $300 \mathrm{~s}$ to exhibit a behavioural response by entering either olfactometer arm. The number of adults entering the treatment arm, control arm or remaining in the inlet adapter (release port) or below T-maze division was recorded. A treatment or control arm choice was recorded when an insect moved into either olfactometer arm by crossing the division in the T-maze olfactometer. A release arm choice was recorded when an insect remained in the release port or below the T-maze division. All experiments were conducted at $26 \pm 1{ }^{\circ} \mathrm{C}$ and $60 \pm 2 \% \mathrm{RH}$. The olfactometer and connecting tubes were thoroughly cleaned with $2 \%$ soap solution and baked at $93.3^{\circ} \mathrm{C}$ between each treatment run. The response of $T$. radiata to Allium spp. volatiles was evaluated following the procedures described for D. citri. However, for this insect only 60 females were tested per treatment combination given that fewer parasitoids were available.

\section{Chemical analysis}

D. citri response to citrus volatiles was inhibited by volatiles from crushed garlic chive essential oil (see results); therefore, the head-space volatiles from these treatments were identified using a static solid phase micro extraction (SPME) technique described in Rouseff et al. (2008). Briefly, a sample was weighed into a $40 \mathrm{ml}$ septum-sealed glass vial. The sample was allowed to equilibrate at ambient laboratory conditions for $\sim 30 \mathrm{~min}$. Accumulated volatiles were collected from the glass vials for $1 \mathrm{~min}$ post exposure to equilibrate the samples to ambient laboratory conditions $\left(20-25^{\circ} \mathrm{C}\right)$. The volatiles were collected using a $75 \mu \mathrm{m}$ carboxen-polydimethylsiloxane (PDMS) Stable Flex ${ }^{\circledR}$ SPME fiber (Supelco, Bellefonte, PA). At least three replicates of each volatile sample were analyzed. The collected volatiles were analyzed and identified using a Perkin/Elmer ${ }^{\circledR}$ Clarus 500 quadrupole mass spectrometer (GC-MS). The GC-MS was equipped with Turbo Mass software (Perkin/Elmer, Shelton, CT) and a $60 \mathrm{~m} \times 0.25 \mathrm{~mm}$, i.d. $\times 0.50 \mu \mathrm{m}$ Restek (RTX-5) capillary column. Helium was used as the carrier gas at a constant flow of $2 \mathrm{ml} \mathrm{min}^{-1}$. The source was kept at $200^{\circ} \mathrm{C}$ and the transfer line and injector were maintained at $260^{\circ} \mathrm{C}$. The oven was programmed from 40 to $260^{\circ} \mathrm{C}$ at $7^{\circ} \mathrm{C} \mathrm{min}{ }^{-1}$. We matched mass spectra with NIST 2005 version 2.0 standard spectra (NIST, Gaithersburg, MD); compounds with spectral fit values $\geqslant 800$ and appropriate LRI values were considered positive identifications. Authentic standards were used to confirm identifications when available.

\section{Behavioural bioassays with synthetic chemicals}

The five sulfur volatiles that were found in greatest abundance in garlic chive essential oil and crushed leaves were tested for repellency to $D$. citri. The bioassay techniques used were similar to those described above. The chemicals were evaluated at $0.25,0.5$ and $1.0 \%$ concentrations individually and in combination with citrus leaves. The chemical samples were dissolved in $1 \mathrm{ml}$ ethylene glycol (EG) and pipetted onto a $5 \mathrm{~cm}$ Richmond cotton wick (Petty John Packaging, Inc., Concord, NC). Given high volatility of sulfur compounds (Dugravot et al., 2004), the synthetic chemicals were dissolved in EG to slow the release rate during bioassays (Arthur, 1996; Dugravot et al., 2002). The

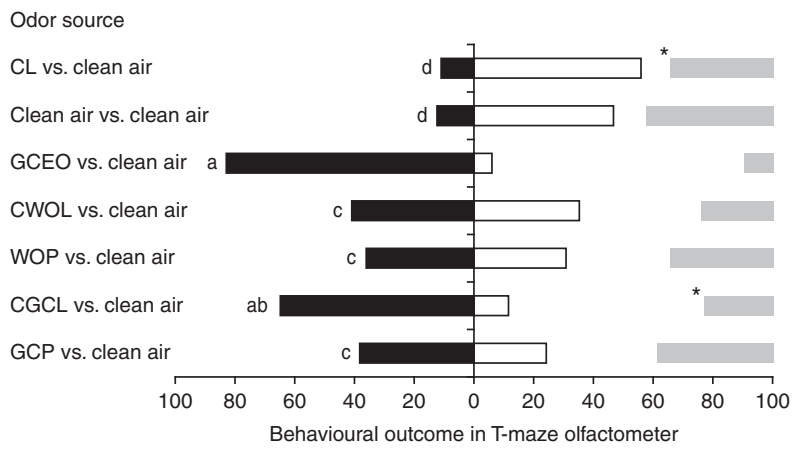

Fig. 2. Responses of Diaphorina citri when presented with volatiles emanating from Allium spp. plants. Garlic chive plant, GCP; crushed garlic chive leaves, CGCL; wild onion plant, WOP; crushed wild onion leaves, CWOL; garlic chive essential oil, GCEO; citrus leaves, CL. Black bars labelled with the same letters are not significantly different (Tukey's HSD TEST, $P<0.05)$. Grey bars labelled with * are significantly different from white bars $\left(\chi^{2}\right.$ test, $\left.P<0.05\right)$ ( $\square$, release arm (\%); $\square$, treatment arm (\%); $\square$, control arm (\%))

treated cotton wick was wrapped in laboratory tissue and placed in solid-phase micro-extraction chambers. The control treatment contained cotton wicks impregnated with $1 \mathrm{ml}$ EG only. For evaluations of chemicals in the presence of citrus odours, $\sim 2.0 \mathrm{~g}$ of fresh citrus leaves were placed in both the chemical treatment and control arm of the olfactometer. The compound showing the highest activity against $D$. citri (dimethyl trisulfide) (DMTS) (see results) was also evaluated as a blend with DMDS at a $0.25 \%$ concentration. DMTS $(0.25 \%)$ vs. clean air was used as the control treatment.

\section{Data analysis}

For assays in which putative repellent treatments were presented in the T-maze olfactometer with or without citrus and vs. clean air, the number of $D$. citri remaining at the release point and not entering the olfactometer was compared between treatments using one way analysis of variance (ANOVA) followed by Tukey's HSD test $(P<0.05)$. For psyllids leaving the release arm, the number of psyllids choosing the control arm vs. the treatment arm was compared with Chi square $\left(\chi^{2}\right)$ analysis at $P<0.05$. The data from all four replicates were combined for the $\chi^{2}$ analysis.

\section{Results}

\section{Behavioural bioassays with plant material}

When clean air was pumped into both arms of the T-maze, the majority of $D$. citri $(89.2 \%)$ left the release arm, indicating a strong negative geotactic and positive phototactic response (fig. 2). Statistically equivalent numbers of $D$. citri entered each arm when clean air was presented vs. clean air, indicating no positional bias. Significantly $(\mathrm{F}=13.98$; $\mathrm{df}=6,21 ; P<0.0001$ ) more $D$. citri did not move from the release point in treatments in which garlic chive odours were co-presented with clean air compared with when citrus vs. clean air or clean air was presented alone (fig. 2). More D. citri remained at the release point when crushed garlic chive leaves or garlic chive essential oil was presented vs. 
clean air than when a whole garlic chive plant vs. clean air, crushed wild onion leaves vs. clean air or a whole wild onion plant vs. clean air were presented (fig. 2). The percentage of $D$. citri not moving from the release point ranged between 65 and $82 \%$ when crushed garlic chive leaves or garlic chive essential oil was presented vs. clean air. When citrus volatiles were released vs. clean air, significantly $\left(\chi^{2}=8.98\right.$; $\mathrm{df}=1 ; P<0.0001)$ more $D$. citri entered the arm receiving citrus odours than clean air (fig. 2). When garlic chive leaf volatiles were presented vs. clean air, significantly $\left(\chi^{2}=5.49\right.$; $\mathrm{df}=1 ; P=0.02$ ) fewer $D$. citri entered the arm with leaf volatiles than the arm receiving the clean air (fig. 2).

Significantly $(\mathrm{F}=6.19 ; \mathrm{df}=3,12 ; P<0.0087)$ more $D$. citri did not move from the release point in treatments in which

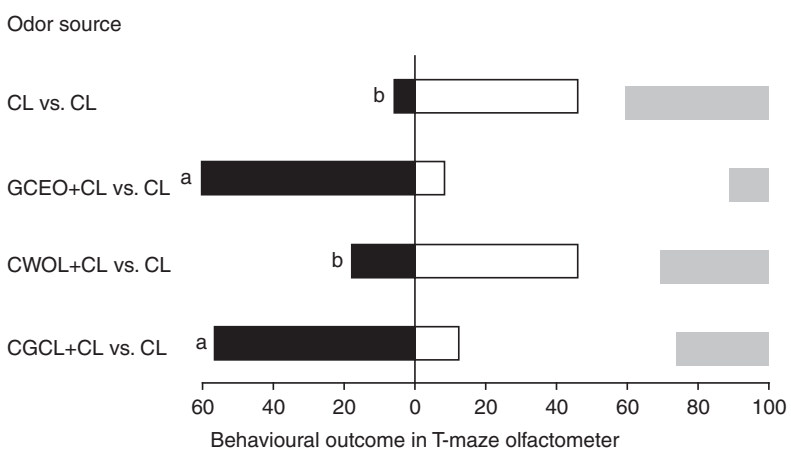

Fig. 3. Responses of Diaphorina citri when presented with volatiles emanating from Allium spp. plants with or without citrus odours. Crushed garlic chive leaves, CGCL; crushed wild onion leaves, CWOL; garlic chive essential oil, GCEO; citrus leaves, CL. Black bars labelled with the same letters are not significantly different (Tukey's HSD TEST, $P<0.05)(\boldsymbol{\square}$, release arm (\%); $\square$, treatment arm (\%); $\square$, control arm (\%)). crushed garlic chive leaves or garlic chive essential oil were co-presented with citrus leaves compared with when citrus leaves vs. citrus leaves were presented (fig. 3). However, the number of $D$. citri choosing each arm of the olfactometer were comparable when crushed garlic chive leaves + citrus leaves or garlic chive essential oil + citrus leaves were presented vs. citrus leaves (fig. 3). Volatiles from crushed garlic chive leaves and garlic chive essential oil did not affect the behaviour of $T$. radiata $(\mathrm{F}=4.69 ; \mathrm{df}=2,5 ; P=0.0713$ ) (fig. 4).

\section{Chemical analysis}

Allyl methyl trisulfide (AMTS), diallyl trisulfide (DATS), DMTS, ADS, AMDS, DMDS, diallyl sulfide (DAS) and AMS were identified from garlic chive essential oil (fig. 5). Five of these sulfur volatiles (AMS, ADS, AMDS, DMDS and DMTS) were also found in the head space of crushed garlic chive leaf samples (data not shown).

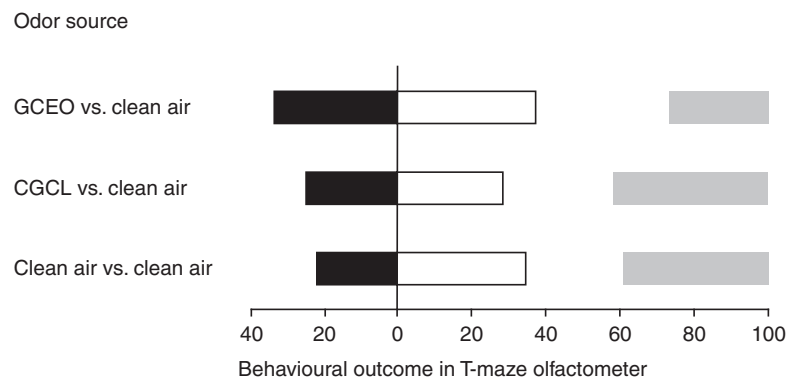

Fig. 4. Responses of Tamarixia radiata when presented with volatiles emanating from Allium spp. plants. Crushed garlic chive leaves, CGCL; crushed wild onion leaves, CWOL. release arm (\%); $\square$, treatment arm (\%); $\square$, control arm (\%)).

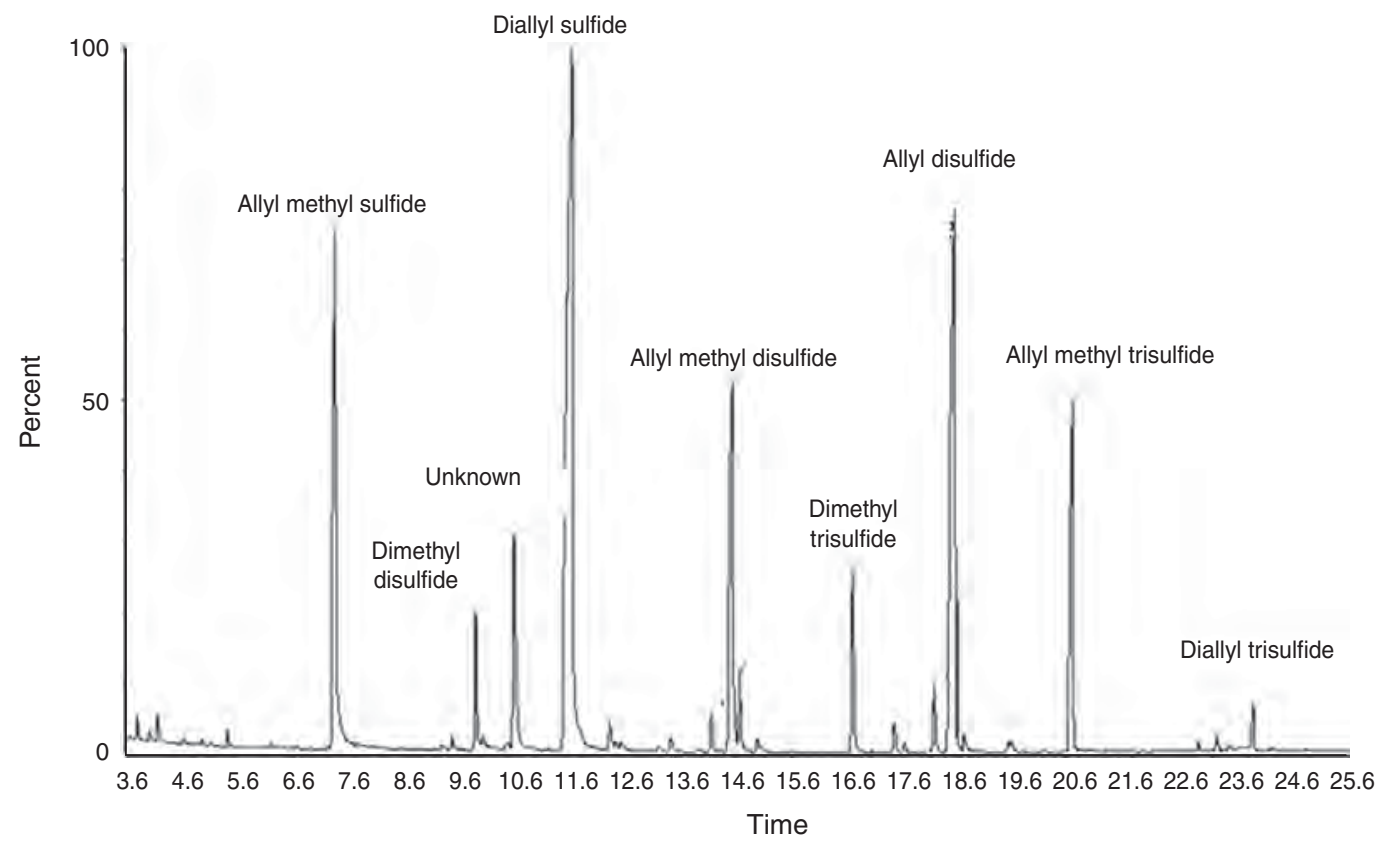

Fig. 5. Example chromatogram showing main sulfur volatiles identified from garlic chive essential oil. 
a

Odor source

Citrus vs. citrus

Clean air vs. clean air

AMS+citrus vs. citrus

AMS vs. clean air

ADS+citrus vs. citrus

ADS vs. clean air

AMDS+citrus vs. citrus

AMDS vs. clean air

DMDS+citrus vs. citrus

DMDS vs. clean air

DMTS+citrus vs. citrus

DMTS vs. clean air

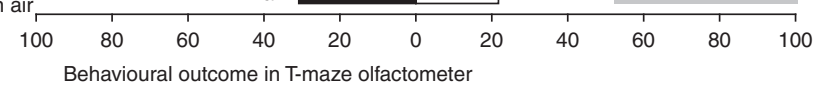

b

Odor source

Citrus vs. citrus

Clean air vs. clean air

AMS+citrus vs. citrus

AMS vs. clean air

ADS+citrus vs. citrus

ADS vs. clean air

AMDS+citrus vs. citrus

AMDS vs. clean air

DMDS+citrus vs. citrus

DMDS vs. clean air

DMTS+citrus vs. citrus

DMTS vs. clean air

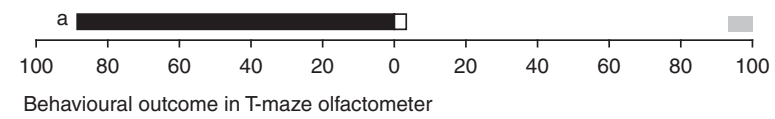

C Odor source

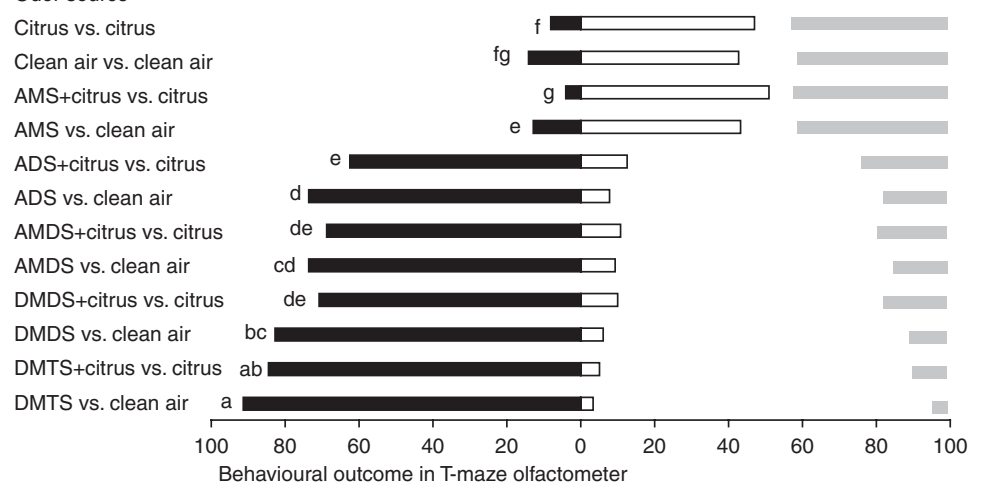

Fig. 6. Responses of Diaphorina citri when presented with sulfur volatiles at (a) $0.25 \%$, (b) $0.5 \%$ or (c) $1.0 \%$ concentration with or without citrus odours. Allyl methyl sulfide, AMS; allyl disulfide, ADS; allyl methyl disulfide, AMDS; dimethyl disulfide, DMDS; dimethyl trisulfide, DMTS. Black bars labelled with the same letters are not significantly different (Tukey's HSD TEST, $P<0.05)(\boldsymbol{\square}$, release arm (\%); $\square$, treatment arm (\%); $\square$, control arm (\%)).

\section{Behavioural bioassays with synthetic chemicals}

Significantly $(\mathrm{F}=9.67 ; \mathrm{df}=11,36 ; P<0.0001)$ more $D$. citri did not move from the release point in treatments in which DMTS (at $0.25 \%$ concentration) was co-presented with clean air or citrus odours than when clean air or citrus were presented simultaneously in both arms of the olfactometer (fig. 6a). No other synthetic sulfur chemical yielded significant differences from the control treatment (clean air vs. clean air) at the $0.25 \%$ concentration with respect to the number of $D$. citri leaving the release point (fig. 6a). Significantly $\left(\chi^{2}=11.58 ; \quad \mathrm{df}=1 ; \quad P=0.0007\right)$ more psyllids entered the control arm than the treatment arm when $0.25 \%$ DMTS was compared with clean air (fig. 6a). 
Significantly $(\mathrm{F}=48.67 ; \mathrm{df}=11,36 ; P<0.0001)$ more $D$. citri did not move from the release point in treatments in which DMTS, DMDS, AMDS or ADS (all at 0.50\%) was copresented with clean air or citrus odours compared with when clean air alone or citrus odour was simultaneously presented in both arms of the olfactometer (fig. 6b). The percentages of psyllids not moving from the release point for DMTS vs. citrus and DMTS vs. clean air ranged between 79 to $89 \%$, respectively, while the percentages of psyllids not moving from point of release for disulfides (DMDS, AMDS and ADS) ranged between 41 and 60\% (fig. 6b). Significantly more $D$. citri chose the arm with clean air compared with $\operatorname{DMDS}\left(\chi^{2}=4.4 ; \mathrm{df}=1 ; P=0.0350\right)$ or $\operatorname{AMDS}\left(\chi^{2}=6.24 ; \mathrm{df}=1\right.$; $P=0.0124)$. Additionally, significantly more $D$. citri chose the arm with citrus odours compared with DMDS $\left(\chi^{2}=4.03\right.$; $\mathrm{df}=1 ; P=0.0447)$ or $\operatorname{AMDS}\left(\chi^{2}=4.79 ; \mathrm{df}=1 ; P=0.0285\right)$ (fig. 6b). The percentages of psyllids moving from the release point to DMTS vs. clean air and DMTS vs. citrus were 11 and $21 \%$, respectively.

Significantly $(\mathrm{F}=114.71 ; \quad \mathrm{df}=11,36 ; \quad P<0.0001) \quad$ more D. citri did not move from the release point in treatments in which DMTS, DMDS, AMDS or ADS (all at 1.0\%) was copresented with clean air or citrus odours than when clean air alone or citrus was simultaneously presented in both arms of the olfactometer (fig. 6c). The percentages of D. citri not moving from the release point for DMTS vs. citrus and DMTS vs. clean air ranged between 84 and 92\%. The percentages of psyllids not moving from release point in treatments with disulfides (DMDS, AMDS and ADS) ranged between 62 and $82 \%$ (fig. 6C). Percentages of D. citri not moving from the release point were statistically equivalent for trisulfides and disulfides (fig. 6c). There were no differences between the number of $D$. citri entering the olfactometer arm containing AMS vs. clean air $\left(\chi^{2}=0.01 ; \mathrm{df}=1\right.$; $P=0.9081)$. Significantly $\left(\chi^{2}=8.92 ; \mathrm{df}=1 ; P=0.0028\right)$ more psyllids remained in the release arm when the $0.25 \%$ blend of DMTS + DMDS vs. clean air was presented than when DMTS alone $(0.25 \%)$ vs. clean air was presented (fig. 7 ).

\section{Discussion}

As expected, more D. citri were attracted to citrus volatiles when citrus leaves were compared with clean air. Furthermore, up to $80 \%$ of tested D. citri entered the olfactometer in response to light and due to negative geotaxis when clean air was presented in both arms. The current results provide evidence that volatiles from crushed garlic chive leaves inhibited the response of $D$. citri to its normally attractive host plant volatiles. These volatiles also appeared to have inhibited the psyllid's normal geotactic and phototactic responses. Furthermore, our results suggest that the sulfur volatiles released by wounded $A$. tuberosum leaves affected the behaviour of $D$. citri. Volatiles released by plants in response to wounding repel herbivoures and deter their feeding (Dicke \& van Loon, 2000; Dugravot et al., 2003). Volatiles from Allium spp. have been previously reported to repel several insect species including hemipterans (Salas, 2001; Assis et al., 2007).

Allium spp. are known to produce sulfur compounds (thiosulfinates) when leaf tissues are wounded. The disulfide and trisulfide breakdown products of the thiosulfinates are toxic to insect herbivoures (Auger et al., 1989). We speculated that the effect of garlic chive plants or essential oil on D. citri may be due to the release of sulfur volatiles given their

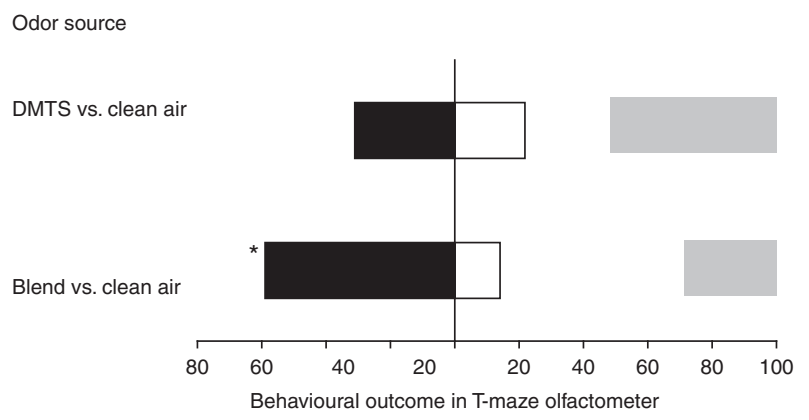

Fig. 7. Responses of Diaphorina citri when presented with a blend of $0.25 \%$ DMTS + DMDS. Dimethyl trisulfide: DMTS. Blend: DMTS $(0.25 \%)+$ dimethyl disulfide $(0.25 \%)$. Black bars labelled with * are significantly different $\left(\chi^{2}\right.$ test, $\left.P<0.05\right)(\square$, release arm (\%); $\square$, treatment arm (\%); $\square$, control arm (\%)).

known effect on insect behaviour (Auger et al., 1989; Huang et al., 2000; Block, 2005; Prowse et al., 2006; Amarawardana et al., 2007; Rouseff et al., 2008). GC-MS analysis of garlic chive essential oil and crushed garlic chive leaves revealed five common sulfur-containing compounds (AMS, ADS, AMDS, DMDS and DMTS). Behavioural assays indicated that trisulfides had a greater effect on $D$. citri behaviour than disulfides and that monosulfides had no effect. Crushed wild onion leaves, containing higher proportions of monosulfides than disulfides and trisulfides, as well as wild onion plants did not affect the response of $D$. citri. Disulfides and trisulfides are known plant defensive chemicals and are toxic to several insect species (Auger et al., 1989; Huang et al., 2000; Dugravot et al., 2005; Rouseff et al., 2008). Furthermore, garlic chive essential oil was more repellent to psyllids than garlic chive or wild onion plants, whether they were mechanically wounded or intact. It is possible that the amount of repellent chemical(s) emanating from the essential oil was greater than that from plants. Future descriptive investigations of the effect of airborne concentrations of these odours on psyllid behaviour will be conducted to resolve the role of concentration versus that of specific chemicals on repulsion of D. citri.

In addition to effects on behaviour, we also observed that D. citri exposed to disulfides and trisulfides died at the $1.0 \%$ exposure concentration. DMDS, AMDS and diallyl trisulfide are known to kill several insect species, including Sitophilus zeamais Motschulsky (Coleoptera: Curculionidae), Tribolium castaneum (Herbst) (Coleoptera: Tenebrionidae) and Periplaneta americana (L.) (Dictyoptera: Blattidae) (Norris et al., 1977; Huang et al., 2000; Dugravot, 2003). Sulfur compounds inhibit chemoreception by destroying sulfur electrochemical receptors (Norris et al., 1977) or interfere with the cytochrome oxidase mitochondrial complex, causing loss of neural activity (Dugravot, 2003). Our results indicate that trisulfides were more potent than disulfides. To our knowledge, there are no studies on the mode of action of trisuflide toxicity to insects. Our results on trisulfides corroborate the findings of Huang et al. (2000) who reported that trisulfides from Allium spp. are more potent insect inhibitors than disulfides.

Both disulfides and trisulfides repelled D. citri vs. clean air at the lower concentrations and inhibited response of pyllids to citrus. However, a blend of dimethyl trisulfide and dimethyl disulfide was more inhibitory than the individual 
constituents, suggesting an additive effect. Our findings are congruent with that of Podskalska et al. (2009) who demonstrated synergistic action of disulfides and trisulfides on Nicrophorus vespillo L. (Coleoptera: Silphidae).

In response to herbivore damage, several plant species emit volatiles that attract natural enemies of herbivores (Arimura et al., 2000). Conversely, induced volatiles may have detrimental effects on natural enemies. Therefore, we also investigated whether garlic chive volatiles affect the behaviour of the $D$. citri parasitoid, T. radiata. There was no effect of these volatiles on the behaviour of the parasitoid, suggesting their potential use for management of $D$. citri should not affect this natural enemy. The current results indicate that disulfides and trisulfides from crushed garlic chive leaves are potential repellents for development and integration into $D$. citri management programmes. Our current efforts are focusing on formulating these sulfur compounds into controlled release devices for deployment in the field.

\section{Acknowledgements}

We thank M. Flores and S. Hermann for their assistance in carrying out bioassays. We also wish to thank Emily Ballentine for drawing the olfactometer diagram. Comments from Dr K. Pelz-Stelinski and two anonymous referees improved a previous version of the manuscript. This research was supported in large part by the Florida Citrus Production Research Advisory Council to L. Stelinski and R. Rouseff.

\section{References}

Amarawardana, L., Bandara, P., Kumar, V., Pettersson, J., Ninkovic, V. \& Glinwood, R. (2007) Olfactory response of Myzus persicae (Homoptera: Aphididae) to volatiles from leek and chive: potential for intercropping with sweet pepper. Acta Agriculturae Scandinavia B 57, 87-91.

Arimura, G., Ozawa, R., Shimoda, T., Nishioka, T., Boland, W. \& Takabayashi J. (2000) Herbivory-induced volatiles elicit defense genes in lima bean leaves. Nature 406, 512-515.

Arthur, F.H. (1996) Grain protectants: current status and prospects for the future. Journal of Stored Product Research 32, 293-302.

Assis, F.A., Moraes, J.C. \& Assis, G.A. (2007) Effect of the aqueous extract of garlic bulbs on the aphid Myzus persicae (Sulzer) (Hemiptera: Aphididae) on potato. Ecossistema 3, 63-66.

Aubert, B. (1990) Integrated activities for the control of huanglongbinggreening and its vector Diaphorina citri Kuwayama in Asia. pp. 133-144 in Aubert, B., Tontyaporn, S. \& Buangsuwon, D. (Eds) Proceedings of the Fourth FAO-UNDP International Asia Pacific Conference on Citrus Rehabilitation, 4-10 February 1990, Chiang Mai, Thailand.

Auger, J., Lecomte, C. \& Thibout, E. (1989) Leek odour analysis by gas chromatography and identification of most active substance for leek moth Acrolipeopsis assectella. Journal of Chemical Ecology 15, 1847-1854.

Beattie, G.A.C., Holford, P., Mabberley, D.J., Haigh, A.M., Bayer, R. \& Broadbent, P. (2006) Aspects and insights of Australia-Asia collaborative research on huanglongbing. pp. 47-64 in Proceedings of the International Workshop for the Prevention of Citrus Greening Disease in Severely Infected
Areas, 7-9 December 2006, International Research Division, Ishigaki, Japan.

Block, E. (2005) Biological activity of Allium compounds: recent results. Acta Horticulture 688, 41-57.

Bové, J.M. (2006) Huanglongbing: a destructive, newlyemerging, century-old disease of citrus. Journal of Plant Pathology 88, 7-37.

Capoor, S.P. (1963) Decline of citrus trees in India. Bulletin of National Institute of Science India 24, 48-64.

Catling, H.D. (1970) Distribution of psyllid vectors of citrus greening disease with notes on the biology and bionomics of Diaphorina citri. FAO Plant Protection Bulletin 18, 8-15.

Childers, C.C. \& Rogers, M.E. (2005) Chemical control and management approaches of the Asian citrus psyllid, Diaphorina citri Kuwayama (Hemiptera: Psyllidae) in Florida citrus. Proceedings of Florida State Horticultural Society 118, 49-53.

Dicke, M. \& van Loon, J.J.A. (2000) Multitrophic effects of herbivore-induced plant volatiles in an evolutionary context. Entomologia Experimentalis et Applicata 97, 237-244.

Dugravot, S., Sanon, A., Thibout, E. \& Huignard, J. (2002) Susceptibility of Callosobruchus maculatus (Coleoptera: Bruchidae) and its parasitoid Dinarmus basalis (Hymenoptera: Pteromalidae) to sulfur-containing compounds: consequences on biological control. Environmental Entomology 31, 550-557.

Dugravot, S., Grolleau, F., Macherel, D., Rochetaing, A., Hue, B., Stankiewicz, M., Huignard, J. \& Lapied, B. (2003) Dimethyl disulfide exerts insecticidal neurotoxicity through mitochondrial dysfunction and activation of insect KATP channels. Journal of Neurophysiology 90, 259-270.

Dugravot, S., Thibout, E., Abo-ghalia, A. \& Huignard, J. (2004) How a specialist and a nonspecialist insect cope with the dimethyl disulfide produced by Allium porrum. Entomologia Experimentalis et Applicata 113, 173-179.

Dugravot, S., Mondy, N., Mandon, N. \& Thibout, E. (2005) Increased sulfur precursors and volatiles production by the leek Allium porrum in response to specialist insect attack. Journal of Chemical Ecology 31, 1573-1561.

Gurusubramanian, G. \& Krishna, S.S. (1996) The effects of exposing eggs of four cotton insect pests to volatiles of Allium sativum (Liliaceae). Bulletin of Entomological Research 86, 29-31.

Halbert, S.E. \& Manjunath, K.L. (2004) Asian citrus psyllids (Sternorrhyncha: Psyllidae) and greening disease of citrus: a literature review and assessment of risk in Florida. Florida Entomologist 87, 330-353.

Hall, D.G. \& Albrigo, L.G. (2007) Estimating the relative abundance of flush shoots in citrus, with implications on monitoring insects associated with flush. Horticulture Science 42, 364-368.

Hall, D.G., Gottwald, T.R., Nguyen, N.C., Ichinose, K., Le, Q.D., Beattie, G.A.C. \& Stover, E. (2008) Greenhouse investigations on the effect of guava on infestations of Asian citrus psyllid in grapefruit. Proceedings of Florida State Horticultural Society 121, 104-109.

Hodges, A.C. \& Morse, J.C. (2009) Southern plant diagnostic network invasive arthropod workshop, May 7-9, 2007. Journal of Insect Science 9, 61.

Hoy, M.A. \& Nguyen, R. (2001) Classical biological control of Asian citrus psylla. Citrus Industry 81, 48-50.

Hrutfiord, B.F., Hopley, S.M. \& Gara, R.I. (1974) Monoterpenes in sitka spruce: within tree and seasonal variation. Phytochemistry 13, 2167-2170. 
Huang, Y., Chen, S.X. \& Ho, S.H. (2000) Bioactivities of methyl allyl disulfide and diallyl trisulfide from essential oil of garlic to two species of stored-product pests, Sitophilus zeamais (Coleoptera: Curculionidae) and Tribolium castaneum (Coleoptera: Tenebrionidae). Journal of Economic Entomology 93, 537-543.

Kristoffersen, L., Hallberg, E., Walle'n, R. \& Anderbrant, O. (2006) Sparse sensilla array on Trioza apicalis (Homoptera: Triozidae) antennae: an adaptation to high stimulus level? Arthropod Structure and Development 35, 85-92.

Landolt, P.J., Hofstetter, R.W. \& Biddick, L.L. (1999) Plant essential oils as arrestants and repellents for neonate larvae of the codling moth (Lepidoptera: Tortricidae). Environmental Entomology 28, 954-960.

Moran, V.C. \& Brown, R.P. (1973) The antennae, host plant chemoreception and probing activity of the citrus psylla, Trioza erytreae (Del Guercio) (Homoptera: Psyllidae). Journal of Entomological Society of South Africa 36, 191-202.

Norris, D.M., Rozental, J.M., Samberg, G. \& Singer, G. (1977) Protein-sulfur dependent differences in the nerve receptors for repellent 1,4-naphthoquinones in two strains of Periplaneta americana. Comparative Biochemistry and Physiology 57, 55-59.

Onagbola, E.A., Boina, D.R., Herman, S.L. \& Stelinski, L.L. (2009) Antennal sensilla of Tamarixia radiata (Hymenoptera: Eulophidae), a parasitoid of Diaphorina citri (Hemiptera: Psyllidae). Annals of Entomological Society of America 102, 523-531.

Podskalska, H., Ruzicka, J., Hoskovec, M. \& Salek, M. (2009) Use of infochemicals to attract carrion beetles into pitfall traps. Entomologia Experimentalis et Applicata 132, 59-64.

Powell, C.A., Burton, M.S., Pelpsi, M.A. \& Bullock, R.C. (2007) Effects of insecticide on Asian citrus psyllid (Hemiptera: Psyllidae) populations in a Florida citrus grove. Plant Health Progress. http://www.plantmanagementnetwork. org/php/ (accessed 24 May 2010).

Prowse, G.M., Galloway, T.S. \& Foggo, A. (2006) Insecticidal activity of garlic juice in two dipteran pests. Agricultural and Forest Entomology 8, 1-6.
Qureshi, J.A. \& Stansly, P.A. (2007) Integrated approaches for managing the Asian citrus psyllid Diaphorina citri (Homoptera: Psylidae) in Florida. Proceedings of Florida State Horticultural Society 120, 110-115.

Qureshi, J.A., Rogers, M.E., Hall, D.G. \& Stansly, P.A. (2009) Incidence of invasive Diaphorina citri (Hemiptera: Psyllidae) and its introduced parasitoid Tamarixia radiata (Hymenoptera: Eulophidae) in Florida citrus. Journal of Economic Entomology 102, 247-256.

Rogers, M.E. (2008) General pest management considerations responses to greening and its vectors have modified pest management programmes. Citrus Industry 89, 12-17.

Rogers, M.E. \& Timmer, L.W. (2007) Florida pest management guide update. Citrus Industry 88, 11-12.

Rouseff, R.L., Onagbola, E.O., Smoot, J.M. \& Stelinski, L.L. (2008) Sulfur volatiles in guava (Psidium guajava L.) leaves: possible defense mechanism. Journal of Agricultural and Food Chemistry 56, 8905-8910.

Salas, J. (2001) Efficacy of a garlic based repellent on the reduction of whitefly (Bemisia tabaci) populations. Agronomia Tropical (Maracay) 51, 163-174.

Snow, K. \& Cutler, R. (2006) A preliminary note on the evaluation of garlic as a mosquito repellent. European Mosquito Bulletin 21, 23-24.

Valerio, L. \& Maroli, M. (2005) Evaluation of repellent and antifeeding effect of garlic oil (Allium sativum) against the bite of phlebotomine sandflies Diptera: Psychodidae. Annali dell'Istituto Superiore di Sanita 41, 243-256.

Wenninger, E.J. \& Hall, D.G. (2007) Daily timing of mating and age at reproductive maturity in Diaphorina citri (Hemiptera: Psyllidae). Florida Entomologist 90, 715-722.

Wenninger, E.J., Stelinski, L.L. \& Hall, D.G. (2009) Roles of olfactory cues, visual cues, and mating status in orientation of Diaphorina citri kuwayama (Hemiptera: Psyllidae) to four different host plants. Environmental Entomology 38, 225-234.

Zaka, S.M., Zeng, X.N., Holford, P. \& Beattie, G.A.C. (2010) Repellent effect of guava leaf volatiles on settlement of adults of citrus psylla, Diaphorina citri Kuwayama, on citrus. Insect Science 17, 39-45. 